\title{
Vorhofflimmern
}

\section{Rasche Kardioversion - auch ohne Schock}

Bei Vorhofflimmern wird zur Wiederherstellung des Sinusrhythmus meist die elektrische Kardioversion bevorzugt. Bei ausgewählten Patienten ist auch die medikamentöse Konversion eine effektive Option.

Die Erwartung, mit Vernakalant (Brinavess ${ }^{\circledR}$ ) die Behandlung bei ausgewählten Patienten mit Vorhofflimmern im oft betriebsamen Alltag einer Notfallabteilung beschleunigen zu können, hat Juhlin und seine Mitarbeiter veranlasst, neben der elektrischen Schockbehandlung auch die Kardioversion mit diesem Antiarrhythmikum in das Programm aufzunehmen.

Im Februar 2011 begann man, Erfahrungen mit der medikamentösen Kardioversion im Klinikalltag zu sammeln - „vorsichtig und Schritt für Schritt", wie Juhlin betonte. In die Auswahl kamen Patienten, die bei der Aufnahme nicht länger als 48 Stunden bestehendes Vorhofflimmern hatten. Sofern eine medikamentöse Kardioversion möglich erschien, wurde Vernakalant $(3 \mathrm{mg} / \mathrm{kg}$ Körpergewicht) in Gegenwart eines Kardiologen und unter EKG-Monitoring über zehn Minuten intravenös verabreicht. War die Kardioversion erfolgreich, wurde der Patient nach zweistündigem EKG-Monitoring entlassen.
Bei weiter bestehendem Vorhofflimmern erfolgte nach 15 Minuten eine zweite zehnminütige Infusion ( $2 \mathrm{mg} / \mathrm{kg}$ Körpergewicht). Blieb auch dieser Versuch erfolglos, konnte nach zwei Stunden eine Elektrokardioversion vorgenommen werden.

Zwischen Februar 2011 und Juli 2012 wurden auf diese Weise 242 Patienten behandelt. Die Dauer des Vorhofflimmerns betrug maximal 24 Stunden (im Schnitt 10,2 Stunden). Bei 167 Patienten (69\%) führte Vernakalant zu einer raschen Konversion im Sinusrhythmus, berichtete Juhlin. Die Zeit bis zur Konversion betrug im Median zehn Minuten.

In den beim Kongress in München vorgestellten neuen "fokussierten" ESC-Leitlinien zum Vorhofflimmern wird Vernakalant erstmals ausführlich gewürdigt. Für Patienten, bei denen eine medikamentöse Kardioversion geplant ist und bei denen keine oder nur eine mäßig ausgeprägte strukturelle Herzerkrankung besteht, wird nun auch die intravenöse Behandlung mit Vernakalant als effektive Option empfohlen. Die Empfehlung gilt auch für Patienten mit postoperativem Vorhofflimmern nach Herzoperationen.

(ob) II

II Satellitensymposium, ESC-Kongress, München, 28. August 2012 (Veranstalter MSD)

\section{Kurz notiert}

Neue Aortenklappe für TAVI

Die Medtronic CoreValve ${ }^{\circledR}$ Evolut $^{\mathrm{TM}}$ 23 Millimeter Aortenklappe hat nun die CE-Kennzeichnung erhalten. Damit bietet Medtronic jetzt vier Klappengrößen an: $23 \mathrm{~mm}, 26 \mathrm{~mm}, 29$ $\mathrm{mm}$ und $31 \mathrm{~mm}$. Die neue 23-mmKlappe verfügt über eine Technologie zur Optimierung der Einpassung, die TruFit ${ }^{\mathrm{TM}}$-Technologie, wodurch die Abdichtung zwischen der Klappenprothese und der Nativklappe unterstützt wird. „Da die CoreValve Evolut-Technologie sich dem anatomischen Sitz anpasst, passt sie sich auch der Anatomie der Patienten an und unterstützt so die Abdichtung", erklärte Prof. Eberhard Grube vom Universitätskrankenhaus Bonn.

II Medtronic

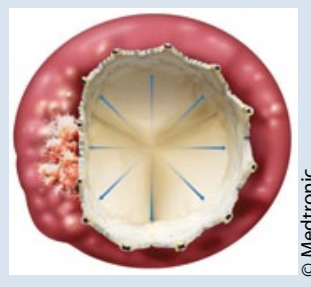

Die Klappenprothese passt sich gut an.

\section{Antihypertensive Therapie}

\section{Es lohnt sich, das Blutdruckziel konsequent zu verfolgen}

Obwohl es in den letzten Jahren gewisse Fortschritte gegeben hat, ist der Blutdruck immer noch bei mehr als $50 \%$ der Hypertoniker nicht ausreichend kontrolliert.

Wie Prof. Vivencio Barrios vom Hospital Ramón y Cajal in Madrid betonte, sind Hochrisikopatienten in einem Kollektiv von Hypertonikern häufiger vertreten als oft angenommen wird. Das gilt nicht nur für spezialisierte Kliniken, wo ihr Anteil 75\% ausmacht, sondern auch für die Primärversorgung mit einem Anteil von $60 \%$.

Die Basis der Therapie für diese Patienten sollte ein Hemmer des Renin-Angiotensin-
Systems (RAS) sein. In der Senkung des kardiovaskulären Risikos sind Angiotensin-IIAntagonisten im Prinzip vergleichbar effektiv wie ACE-Hemmer. Doch die Therapie mit ACE-Hemmern wird aus Verträglichkeitsgründen häufiger abgebrochen, was sich durchaus als relevant erwiesen hat.

Doch fast alle Hypertoniker brauchen mindestens zwei Medikamente, um die Blutdruckzielwerte zu erreichen. In der TRINITYStudie hat sich der Wechsel auf eine Dreifachkombination aus Olmesartan, Amlodipin und Hydrochlorothiazid (als fixe Kombination z.B. als Vocado ${ }^{\oplus} \mathrm{HCT}$ erhältlich) nach vier Wochen Therapie mit einer der drei möglichen Zweifachkombinationen aus diesen
Substanzen gelohnt: Der Blutdruck nahm bis Woche 12 schneller und stärker ab als in den Gruppen, die die Zweifachkombination beibehielten.

Die BP-CRUSH-Studie hat gezeigt, dass sich mit einer am Zielwert orientierten stufenweisen Hochtitration der Medikation (beginnend mit $20 \mathrm{mg}$ Olmesartan und $5 \mathrm{mg}$ Amlodipin, endend mit $40 \mathrm{mg}$ Olmesartan, $10 \mathrm{mg}$ Amlodipin und $25 \mathrm{mg}$ Hydrochlorothiazid) schließlich bei $90 \%$ der Patienten das Blutdruckziel erreichen lässt.

(abi)

II Symposium beim ESC-Kongress, München, 26. August 2012 (Veranstalter: BerlinChemie) 